This item was submitted to Loughborough's Research Repository by the author.

Items in Figshare are protected by copyright, with all rights reserved, unless otherwise indicated.

\title{
Feasibility and acceptability evaluation of the Promoting Independence in Dementia (PRIDE) intervention for living well with dementia
}

\section{PLEASE CITE THE PUBLISHED VERSION}

https://doi.org/10.1017/S1041610220001386

\section{PUBLISHER}

Cambridge University Press

VERSION

AM (Accepted Manuscript)

\section{PUBLISHER STATEMENT}

This article has been published in a revised form in International Psychogeriatrics http://doi.org/10.1017/S1041610220001386. This version is published under a Creative Commons CC-BYNC-ND. No commercial re-distribution or re-use allowed. Derivative works cannot be distributed. () International Psychogeriatric Association.

\section{LICENCE}

CC BY-NC-ND 4.0

\section{REPOSITORY RECORD}

Csipke, Emese, Esme Moniz-Cook, Phuong Leung, Lauren Yates, Linda Birt, Holly Walton, Eef Hogervorst, Gail Mountain, Georgina Charlesworth, and Martin Orrell. 2020. "Feasibility and Acceptability Evaluation of the Promoting Independence in Dementia (PRIDE) Intervention for Living Well with Dementia". Loughborough University. https://hdl.handle.net/2134/13668833.v1. 
Promoting Independence in Dementia (PRIDE): Feasibility trial of the PRIDE social intervention for living well and independently with mild dementia

Dr Emese Csipke ${ }^{1^{*}}$

Professor Esme Moniz-Cook ${ }^{2}$

Dr Phuong Leung ${ }^{1}$

Dr Lauren Yates ${ }^{3}$

Dr Linda Birt ${ }^{4}$

Dr Holly Walton ${ }^{5}$

Professor Eef Hogervorst ${ }^{6}$

Professor Gail Mountain ${ }^{7}$

Dr Georgina Charlesworth ${ }^{8}$

Professor Martin Orrell ${ }^{3}$

*corresponding author

${ }^{1}$ Division of Psychiatry, University College London, UK

${ }^{2}$ Faculty of Health Sciences, University of Hull, Hull, UK

${ }^{3}$ Institute of Mental Health, University of Nottingham, UK

${ }^{4}$ Faculty of Medicine and Health Sciences, University of East Anglia UK

${ }^{5}$ National Centre for Sports and Exercise Medicine, Loughborough University, Loughborough UK

${ }^{6}$ Department of Applied Health Research, University College London, London, UK

${ }^{7}$ Centre for Applied Dementia Studies, University of Bradford, Bradford UK

${ }^{8}$ Clinical Educational and Health Psychology, University College London, London UK

Contact details: e.csipke@ucl.ac.uk*

Key words:

Dementia, Independence, Decision-making, Feasibility, Post-diagnostic support, Intervention 


\section{ABSTRACT}

Background: Post diagnostic psychosocial interventions can play an important role in supporting persons with mild dementia to remain independent and at home for longer. The PRIDE intervention was developed to address this growing need. We tested the feasibility and acceptability of this facilitator led psychosocial intervention in the community.

Method: This mixed methods, non randomised, pre-post feasibility trial was run across four sites across England. Facilitators were recruited from the voluntary sector and memory services. Participants with dementia and their supporters took part in the 3 session intervention. Outcome measures were collected at baseline and at follow up, and a subsample of participants took part in focus groups and interviews following completion of the trial to assess acceptability.

Results: Thirty four dyads consented to be in the study over a recruitment period of ten months, with 14 facilitators providing the itnervention. $79 \%$ of dyads took part in at least two sessions, and $73 \%$ in all three. Outcome measures were completed by $79 \%$ of participants without difficulty, and with minimal missing data. No significant changes were found on pre and post assessments, but modest effect sizes were. Qualitative data indicated that dyads found the intervention acceptable, as did intervention facilitators.

Conclusions: Our psychosocial intervention designed to assist people with dementia maintain independence and remain socially, cognitively and physically active was successfully delivered. Preliminary findings indicate that the intervention is acceptable and further testing feasible and It will be worthwhile to evaluate the PRIDE intervention in a large randomised controlled trial.

Key words: Feasibility, acceptability, dementia, memory services, psychosocial, self management 


\section{INTRODUCTION}

The EU Joint Programme for Neurodegenerative Disease Research and the UK government have both highlighted the need for the development of high quality specialist services, in particular the provision of psychosocial interventions to support the growing needs of people with dementia (Challenge on Dementia 2020 - Department of Health 2016; EU JPND 2012). People with dementia may reduce their activities due to perceived stigma, neurodegenerative decline, and loss of autonomy and self-confidence (Birt, Poland, Csipke \& Charlesworth, 2017; Lion, et al 2019). Furthermore, family and friends may behave in over protective ways and undermine the selfdetermination and independence people with dementia may wish for (Sterin, 2002). However, receipt of support soon after diagnosis may facilitate better adjustment and ongoing management of dementia (Burgener, Buettner, Beattie \& Rose, 2009), and can help people remain more independent and part of their community, while delaying residential home placement (Clarke et al., 2013).

The Promoting Independence in Dementia (PRIDE) research programme seeks to help improve independence post diagnosis by delivering a psychosocial intervention for those with mild dementia. The development of this brief intervention for people in the mild stages of dementia drew on interconnecting strands of the overall PRIDE project, including epidemiological and qualitative data, expert consensus and PPI involvement (Csipke et al. 2018; Yates et al. 2019). The intervention drew on work from the English Longitudinal Study of Aging (ELSA; $n>10,000$; Steptoe et al, 2013) cohort study which found that loneliness was linked to cognitive decline, although social isolation in itself was not (Rafnsson et al, 2017). Likewise, those who had close social networks had a reduced risk (Khondoker et al, 2017). Our work has also found that digital literacy, computer use and physical activity were associated with lower risk of dementia (D'Orsi et al, 2017; Soni et al, 2017; Stock et al, 2015). A qualitative critique of literature about social participation in dementia highlighted challenges associated with memory loss, social relationships and social structures in enabling people with dementia to remain independent (Birt et al., 2019). The PRIDE intervention aimed to maintain or improve independence in people with dementia in early stage dementia (Yates et al., 2019). Stakeholders (persons with dementia, carers, and older people) contributed to shaping the intervention which included strategies to help people continue to make everyday decisions and adjust to a diagnosis. Unlike many other such interventions, stakeholders were involved throughout the development process.

Feasibility testing can identify potential difficulties with intervention acceptability, recruitment and retention rates, and adherence. The Medical Research Council (MRC) recommends that uncertainties associated with conducting a large scale study can be addressed in feasibility work (Craig, 2008), and preliminary questions should be examined using both qualitative and quantitative methods. The aim of the present study is therefore to examine the feasibility of the PRIDE intervention by investigating: recruitment rate of sites and intervention providers, and participants; the acceptability of, and engagement with the PRIDE intervention; and the acceptability and feasibility of outcome measures.

\section{METHOD}

\section{Design}

This is a mixed methods pre-post feasibility study of the PRIDE intervention (Csipke et al., 2018). Outcome measures were collected at baseline and post-intervention delivery (up to 3 months), to address the study questions. A sub-sample of people with dementia (those who expressed an interest), their supporters and intervention facilitators took part in a qualitative interviews following the intervention to examine acceptability and engagement with PRIDE. 


\section{Participants}

All participants were adults $(18+)$ who were able to read and communicate verbally in English and have the capacity to give informed consent in accordance with the Mental Capacity Act (DOH, 2005).

Persons with dementia were community dwelling with a diagnosis of mild dementia, as defined by 0.5-1 on the Clinical Dementia Rating (CDR) Scale (Morris 1993). They were expected to have a supporter willing to participate with them. Supporters were included since although the intervention is aimed at those with dementia, many of the themes of PRIDE (e.g, decision making, communication skills) take place within a social context. The term supporter was used throughout the study since it denotes support as part of the reciprocity in a relationship, rather than carer which implies dependence.

Supporters (carers) were eligible if they were in regular unpaid contact with the person with dementia (minimum three hours a week).

Referral sources, along with reasons for ineligibility and retention rates were recorded. In between session support (telephone calls, extra sessions) requested by the dyad, as well as that required by sites and intervention facilitators (site visits, further training) were recorded.

\section{Procedures}

Recruitment was through NHS memory services, Join Dementia Research (a national register of people interested in taking part in dementia research), and voluntary sector organisations. Potential participants were approached by healthcare professionals working within these organisations or the study team and given study information if they expressed an interest. If they agreed to participate, researchers arranged to visit them in their homes and sought written informed consent and complete measures. Dyads were linked with local Intervention facilitators who would meet with the dyad on three occasions, approximately four weeks apart. After the final session, follow up assessments were arranged with researchers.

\section{Ethics}

The study was approved by the East Midlands Nottingham 1 Research Ethics Committee (REC; ref no 16/EM/0044). Participants were fully informed of potential risks and benefits of the study and that they would be free to withdraw at any time without affecting their care. Participants with mild dementia were expected to have capacity to provide consent for themselves, which was an ongoing process. The study also obtained Health Research Authority (HRA;

https://www.hra.nhs.uk/approvals-amendments/) approval via the sponsoring university for governance and legal compliance to enable sites to carry out studies. Both ethics and HRS approval are given by independent bodies and required before potential participants can be approached.

\section{Sample size}

No formal sample size calculations were made. However, it was decided that use of geographically spread sites would increase generalisability. The aim was to recruit up to five sites across England, with approximately ten dyads at each site. This would provide sufficient data to evaluate recruitment and withdrawal, whether the recruited participants and the facilitators found the intervention to be acceptable and feasible. Recruitment lasted 10 months.

\section{Sites}

Sites were eligible if they had local researchers to undertake the baseline and follow up measures, could provide a supervising principal investigator; and had access to facilitators to deliver the intervention. Site based researchers were provided with training on screening, measure completion 
and all study procedures by the research team. Likewise, facilitators at each site were provided with training to provide the intervention.

Intervention facilitators were either voluntary sector staff working as dementia advisors providing one on one information, advice and support)alongside or with memory clinics, or health and research staff working with memory services. Service managers referred suitable staff who attended a day long training session and could deliver the intervention .

\section{The PRIDE intervention}

The intervention is delivered by a facilitator at the person's home across three, $60-90$ minute sessions. The intervention aims to equip the person to participate in activities, build on communication skills and to enable them to continue making choices as the dementia progresses. In between sessions the person is encouraged to engage in activities that will support their independence, social inclusion, and engagement in the community (e.g. joining a walking group). This process is supported by a paper based manual that is followed in the sessions, provides a place for written records, and acts as a reference and workbook between sessions. For further details about the PRIDE intervention, theoretical underpinnings, and materials, please refer to Yates et al., 2019.

\section{Acceptability of intervention - qualitative evaluation}

Qualitative data were audio recorded and transcribed. Framework analysis on post intervention focus groups with a convenience sample of participants were planned to determine if; (1) participants found the intervention and study procedures suitable and feasible, and (2) obtain their views on taking part. Intervention facilitators were also asked take part in separate focus groups to obtain their views of the intervention. Focus groups provide an opportunity for new perspectives on experiences to develop as participants discuss and challenge each other's views (Jenson \& Laurie, 2016).

Four men with dementia, their spousal supporters and three intervention facilitators took part in the qualitative phase of the evaluation. The interview topic guides asked participants to consider what they thought and felt about the intervention, the presentation and content of the manual and they were asked to discuss their experience of using it, the relationship between the person with dementia and their supporter, as well as with the intervention.

\section{Outcome Measures}

Measures were chosen to assess the overarching themes of self-management, independence, hope, resilience and social support (the core of the intervention) and economic costs. Measures were read out to participants to ensure consistency and promote inclusiveness for participants who found reading text difficult. Supporters completed their own measures. Measures were either validated tools, or developed and tested in/for PRIDE.

Clinical Dementia Rating (CDR; Morris, 1993): The CDR rates impairment in each of six cognitive categories (Memory, Orientation, Judgment and Problem Solving, Community Affairs, Home and Hobbies, and Personal Care) on a five-point scale.

The Bristol Activities of Daily Living Scale (BADLS; Bucks, Ashworth, Wilcock, \& Siegfried, 1996): The BADLS is a carer rated instrument consisting of 20 daily-living abilities.

Dementia Quality of Life measure (DEMQOL; Smith et al., 2005): The DEMQOL covers five domains of quality of life: health and well-being, cognitive functioning, social relationships and self-concept. 
Control, autonomy, pleasure, and self-realization. (CASP-19; Hyde, Wiggins, Higgs, \& Blane, 2003): The CASP-19 measures quality of life in older people in four domains: control, autonomy, pleasure and self-realisation.

Timed up and go test (Podsiadlo \& Richardson, 1991): Timed up and go test quantifies functional mobility. The test is quick, requires no special equipment or training, and is easily included as part of the routine medical examination.

ELSA social support questions. ELSA uses the short (three item) form of the revised version of the UCLA Loneliness Scale (Hughes, Waite, Hawkley, \& Cacioppo, 2004) which is a short scale for measuring loneliness in large surveys.

Hopkins Verbal Fluency and Learning Test (Hogervorst et al, 2002). The HVLT consists of a 12-item word list, composed of four words from each of three frequently printed semantic categories (e.g. 'animals') which participants are asked to recall. Additionally, participants are asked to name as many animals as they can think of in one minute.

Standardised Mini-Mental State Examination (SMMSE; Vertesi, et al., 2001). Assesses orientation, repetition, verbal recall, attention and calculation, language and visual construction.

Impact on participation and autonomy (IPA; Hammar et al., 2014): The IPA is used when planning healthcare and rehabilitation to ensure their right to self-determination and participation. Only the six items from the social support/relationships subscale were used.

Self-management abilities (SMAS-30; Schuurmans et al., 2005): The SMAS consists of six subscales including taking inititive, variety, self-efficacy, investment behavior, positive frame of mind, and multifunctionality.

ICECAP-O (Coast et al., 2008): ICECAP-O measures five domains include attachment, security, role enjoyment and control.

Health-related quality of life (EQ-5D) (EuroQoL Group, 1990): The EQ-5D is a measure of selfreported health outcomes. Part I of the scale consists of five single-item dimensions including: mobility, self-care, usual activities, pain/discomfort, and anxiety/depression. Part II uses a vertical graduated VAS ( 0 to 100 ) to measure health status, ranging from worst imaginable health state to best imaginable health state.

The Positive Psychology Outcome Measure (PPOM; Stoner et al., 2017). The PPOM is 16 item measure used to assess levels of hope and resilience in people with dementia.

Engagement and Independence in Dementia Questionnaire (EID; Stoner et al., 2017). The EID-Q assesses levels of subjective independence and social engagement.

Fidelity (Walton,2018). Intervention providers and participants were asked to complete fidelity checklists after each visit. The participant checklist included four items on engagement: two of these measure whether the participant understood the information provided to them (receipt) and two of them measured whether the participant enacted the plans (enactment). Engagement questions were rated on a three-point scale: 'yes', 'to some extent', and 'no'. Questions on enactment were completed after the second and third sessions. In this paper, we used the enactment questions as a basic measure of engagement. Full details of the fidelity and engagement are reported elsewhere (Walton, 2018).

Outcome measures for supporters 
Outcome measures for supporters included the Health-related quality of life (EQ5-D; (EuroQoL Group, 1990) and the ICECAP-O (Coast et al., 2008), as described above.

\section{Cost-data}

Client Services Receipt Inventory (CSRI) (Beecham et al., 1992): CSRI is used to collect information on the identified supporter's costs and participant's use of health and social care services. The measure was included to assess the acceptability of its use by participants and was not analysed.

\section{Analyses}

\section{Quantitative}

Researchers completed measures with participants, and difficulties noted. Missing data was examined. Basic pre-post $t$-tests were run. Further post hoc analyses were carried out when differences in means warranted.

\section{Qualitative}

Qualitative data were audio recorded and transcribed. Framework analysis enables an evaluation of applied interventions (Srivasta \& Thomson, 2009). The analytical steps were: familiarisation with the data, generating initial codes which reflected the research questions in topic guides, producing a thematic framework, index charting and mapping of data which enabled reflection on differences and similarities in experiences of stakeholder, Interpretation and presentation of themes. Results were explicitly explored to see if there were differences in the experiences of invention facilitators, supports or people with dementia as this would add further understanding to the efficacy of the intervention.

\section{RESULTS}

\section{Site Recruitment and Set up}

Six NHS Foundation Trusts with memory services across England expressed an interest in participating in the study. Two sites dropped out after not being able to identify both researchers and intervention facilitators who had the capacity to carry out assessments and deliver the intervention. All participating sites were predominantly urban. Each site was provided a site set up visit lasting 2-3 hours, which included training and information covering participant identifying/screening/consenting, and measure administration. Sites were also given instruction in study procedures such as communications with the research team, data collection and entry, and documentation and site files.

Running concurrently with site selection and set up was HRA approval. At the time, the HRA was undergoing a restructuring of the processes and requirements of this approval. This led to considerable delays and took a total of 10 months until it was granted, which considerably delayed the start of participant recruitment. Consequently, as there was no room left for an extension of the recruitment period, the final sample size was smaller than expected.

\section{Intervention facilitator recruitment and Training}

Management staff at voluntary organisations and NHS sites were approached to identify potential intervention providers. Dementia Advisors in the voluntary sector were scarce and contracts were subject to change on a yearly basis, in particular it was difficult for them to participate as the work was not specifically funded or commissioned. Potential facilitators from the voluntary sector were approached early in the planning stages, but due to delays in HRA and site approval many who 
expressed an interest were no longer able to participate as facilitators. Therefore NHS and clinical research staff were also approached. One of the two sites dropped out at a late stage after the intervention provider (voluntary sector) identified was no longer available and a suitable replacement could not be identified quickly. Further challenges were found in that geographically, the research sites did not always match the geographical remit of the intervention providers. Finally, establishing a legal agreement with one of voluntary organisations was very time consuming and led to a delay between local staff remaining available to provide the intervention and the head office signing off on approval.

Twenty-six health professionals from voluntary organisations and NHS teams (clinical and research) registered for and completed the training programme, with sessions delivered at each site. Eleven (43\%) were voluntary sector staff, seven (27\%) dementia researchers, four (15\%) dementia nurses, and four (15\%) allied health professionals. Fifty four percent of those who took part delivered the PRIDE intervention. The remaining 12 trainees (46\%; all voluntary sector) did not deliver the intervention due to high workloads, were managers attending the training for information purposes only, or staff leaving the organisations. Managers and staff not planning to deliver the intervention were invited to attend in order to be able to provide support during the trial, and to maximise interaction opportunities between different teams and build relationships during the training itself.

\section{Dyad recruitment}

One hundred and fifteen potential participants were identified across the four participating sites. The JDR register provided 61 potential participants, the voluntary sector five, 23 participants taking part in other studies expressed an interest, while memory clinics referred 25 , and one was referred by a psychiatrist.

Of these, we were unable to contact $23(20 \%)$, and another $23(20 \%)$ were out of the catchment areas, seven (6\%) were not clinically eligible, $14(12 \%)$ were not eligible for other reasons. Of the 48 remaining eligible, 14 (41\%) declined to take part, leaving 34 dyads recruited to the study.

Recruitment ended at ten months, to allow for time for intervention delivery and follow ups to be completed in time for the next study to start.

The mean age of participants was $77.67(\mathrm{SD}=9.07)$ and $23(67 \%)$ were men. Thirty $(88 \%)$ were White British and four (12\%) were of other ethnicity. Half of participants were diagnosed with Alzheimer's, six (18\%) had mixed dementia, four (12\%) vascular dementias and the remaining seven $(8 \%)$ had an unknown or other dementia diagnosis. Twenty seven (79\%) of supporters were women, with an average age of 69.18 (SD=11.89). Thirty two (94\%) were White British, and two (6\%) were of other ethnicity.

For a flowchart of study recruitment and retention please see Figure 1.

- Insert Figure 1 here -

Four participant/supporter dyads (4 men with dementia; 4 spousal supporters) were recruited to a focus group, at two sites, three intervention providers (all women) agreed to take part in a focus groups. However, it was not possible to coordinate schedules and locations so individual telephone interviews were undertaken.

\section{Intervention Delivery and Uptake}

Of the 34 dyads consenting to take part, 33 took part in the first session. Of these, 26 (79\%) took part in two sessions, and 24 (73\%) in all three sessions. Overall, seven individuals dropped out; one due to ill health, one due to supporter's ill health, one due to the facilitator finding alternate employment (dyad dropped out rather than start with new facilitator) and four gave no reason. No participants requested an extra session. Five individuals took up the offer of telephone contact 
between sessions, with eight calls between them. Calls took 5-15 minutes and involved updates from the participants about their activities since the session prior.

One site reported three SAE's and in all cases it was hospital treatment for a medical condition unrelated to PRIDE. No deaths were reported during the study. No participants moved into supported accommodation or care homes.

Site and intervention providers were offered support from the study team as needed and newsletters updating on progress were sent to each site. The study team offered to provided any additional site visits if needed, only one site took up the offer. All sites had questions about procedural issues which were resolved via telephone or email. Additionally, a group of intervention providers requested a monthly teleconference, but after the initial one they decided they didn't need it after all.

\section{Measure Performance}

Of the dyads who consented to take part, 34 completed the baseline assessment and 27 the follow ups (79\%).

\section{Screening Tool}

Based on the belief that memory clinics routinely used the CDR and would therefore have records of scores, the protocol stated that it would be used as a screening tool. However, many of the memory clinics did not use the CDR. Although training was offered, completion of the CDR would have required the participant to complete a pre-screening consent form (which would have required an ethical amendment). In the interest of not delaying further, an alternative was found. The sites did use the Montreal Cognitive Assessment Test for Dementia (MoCA; Nasreddine et al, 2005) therefore We used this as an alternative screening tool.

\section{Outcome Measures}

Measures were completed without significant difficulty by participants, took approximately 60-90 minutes, and all were completed in one single session. The TUG (which involves walking across a room in straight line) posed some challenges in that some participants' homes did not have much free space that was free of trip hazards such as rugs. But in all cases, this was overcome. Several participants questioned the need for this assessment. None of the measures caused distress.

There were no measures or specific items with enough missing data to warrant concern, and overall less than $2 \%$ of data was missing (over both time points, for both participants and supporters). Following this, multiple imputations with a linear regression were carried out for scale variables at baseline and post intervention in order to impute missing variables.

Exploratory t-tests (baseline versus follow up) were run as per study protocol, however no significant differences were found (Table 1 and 2). Post hoc analysis of effect size found that the SMAS had moderate effect size (d) of.41 indicating follow up scores improved. In addition, the EQ5D-VAS for supporters indicated a moderate effect size (d) of .41, also indicating scores improved. Cost data (CSRI) was not analysed, but it was found that participants were able to complete it.

Further post hoc exploration (effect sizes; Cohen's D) of SMAS scores were carried out taking into consideration engagement with the intervention. In this analysis, only scores on enactment were used (Walton, 2018). Scores were converted into categories of $\leq 75 \%$ or $76-100 \%$ enactment based on the distribution. SMAS means were calculated for the two categories (plus an 'unknown' group of participants who did not complete the checklists) at baselines and follow up. Those with higher engagement scores demonstrated better self-management abilities at baseline, which remained true at follow up (no change in scores). The group with lower engagement also had the lower baseline 
self-management scores, but by the end of the intervention at follow up matched the first group. (Table 3).

(Tables 1, 2 and 3here)

\section{Qualitative results}

Three themes emerged from analysis: understanding the ethos of the PRIDE intervention; relationships within the PRIDE intervention and the relevance of the PRIDE intervention.

\section{Understanding the PRIDE study ethos}

All participants were able to articulate the ethos of the PRIDE intervention as being that of enabling people with dementia to maintain some independence. The sense of hope instilled through the intervention was captured by a facilitator who stated:

It's for maintaining independence, isn't it, and it's ensuring that people are constantly putting things in place to make sure that they live happy, independent and active lives... to stay in control of their lives (Facilitator 2)

People with dementia and their supporters spoke of the confidence they gained through being part of the PRIDE intervention.

thought it was very good. [filling in manual] It gives everybody, erm... can't think of the word... researchers and everybody knows what I'm doing and how I'm thinking. I thought that was good, anyway. (Person with dementia 3)

Facilitators helped participants engage in the intervention. For example where a person with dementia found it difficult to explain their wants the use of a singing video helped her relax and the facilitator noted :

she held my hand and said thank you, she was understanding that we were adapting it not to make her feel threatened or anything like that but to do it to the way that she enjoyed ... so that she could still take part in everything (Facilitator 3)

\section{Relationships within the PRIDE intervention}

The intervention facilitator worked through the manual and activities with the person with dementia, and if they wished their supporter. The facilitator helped the person set priorities and action plans. The face-to-face relationship lasted three sessions and was occasionally supplemented with follow up telephone call. All people spoke of this personal contact as important:

The best benefit we got was actually talking to the people that came out. I think that made a lot of difference, person to person. (Supporter 2)

Both facilitators and supporters acknowledged the essential role the supporter played in encouraging the person with dementia to engage in the intervention. This might have been in following up on action plans and using the manual between sessions

I think only if the supporter was encouraging them - Facilitator

The supporter also helped the person with dementia engage in intervention plans in practical ways such as getting equipment and keeping action plans up to date. 
I think he was definitely prompting her to do the knitting and he went out to the shops and bought the wool with her, and... so yeah, and all the sheets that were filled in, he filled those in for her. But she did it all, she was doing all the activity (Facilitator 1)

However, one facilitator explained how they had had to manage a supporter who wanted to take the lead:

The partner will want to take the lead and speak for the person; we had to change it a little bit just to say please could they [person with dementia] go first and express their feelings (Facilitator 3)

Not all people with dementia had active support from their family; this was reported as being due to poor health of the supporter or limited interested from the wider family.

\section{Relevance of the PRIDE intervention}

The PRIDE intervention was aimed at those with mild dementia and all thought the intervention would be most useful soon after diagnosis. This support explained it helped to reduce fear following a diagnosis:

We wouldn't have known where to go and that actually you can have some sort of life, because when something like that is said to you, you think that's the end. That's actually where the fear comes because really don't know where to turn or what to do and somebody coming in and talking to you about it and helping with these sort of things gives you a vision. (Supporter 4)

I think it's good just being diagnosed because it gives you a look into what help you can get and things like that (Person with dementia 2)

A facilitator confirmed that the PRIDE intervention would give hope to those newly diagnosed with dementia:

I feel that they're trying to recognise somebody that's newly diagnosed, to actually state that because you have a diagnosis, it doesn't mean that life stops. (Facilitator 3)

This contrasted with the other dyads where the person had been living with dementia over 12 months. Here facilitators found it most challenging when they were delivering the intervention to 'socially active' couples; in part because they were already doing activities they enjoyed and also because there were long periods between sessions as dyads took holidays. However, it was also recognised that PRIDE may be suitable to lay the foundations of skills that may be useful as dementia progresses,

Because I do think the lady maybe she is at risk of maybe losing some of those activities' (Facilitator 3).

The intervention delivery was centred on the PRIDE manual (Yates et al, 2019). While the majority found the manual easy to use there were some negative comments on layout and the use of smiley faces, dementia 3).

'If you don't mind me saying, I think this is a kiddie's way of doing this.' (Person with

The facilitators used the manual in every session. However, they all gave examples of how they had had to adapt the delivery; often this was due to over enthusiasm:

On one occasion I had to scrap everything and pull it right back, because we thought too big, it is good experience (Facilitator 1). 


\section{Discussion}

Overall, the intervention and training was feasible to deliver across various sites. The study was well received by those who took part in the sessions. Of those who eligible $59 \%$ consented to be in the study. Seventy nine percent attended at least two sessions, and $73 \%$ attended all three. All 34 completed the baseline measures, and $79 \%$ the follow up. No measures posed significant problems, and there was little missing data, although significant differences were not detected, possibly due to small sample size. Setting up sites to be ready to provide the intervention had its challenges but these were overcome.

Recruiting, sites as well as participants, is one of the common challenges of health research, particularly in multi-site trials, but also the key to their success (Borschmann et al., 2014; Kaur et al, 2012; Nuno et la., 2017). Estimating adequate recruitment timelines for a large trial is one of the key functions of a feasibility study (Thebane et al, 2010). We found two significant obstacles. The HRA approval system was beyond the team's control and unexpected, especially as we had completed a similar procedure some months prior. Secondly, matching available intervention facilitators to research sites was difficult. The feasibility study required the recruitment of sites that not only had the capacity to identify, recruit and assess participants, but to also have available suitably qualified staff to provide the intervention, either in partnership with a voluntary organisation or working with their own NHS team. Initially, it was envisaged that Dementia Advisors from voluntary organisations would be able to fulfil this role (Alzheimer's Society, 2016). Although the project had the support of the head and regional offices of these voluntary organisations we found that it was untenable to rely solely on the voluntary sector. Attrition due to organisational factors and delays in participants recruitment have been found in other studies relying on interventions being delivered by the voluntary sector (Mountain et al. 2017) and although our intervention providers were paid employees, similar issues arose. Therefore, NHS staff working with memory clinics, typically nursing staff and clinical dementia researchers, were approached to facilitate the intervention. This indicates that a wide variety of staff could provide it. Furthermore, we have now developed more specific site requirements when engaging sites on the upcoming larger scale project; sites must identify multiple researchers and intervention facilitators before joining the study. Ellwood et al (2018) also found that a targeted approach to site (in their case, care homes) recruitment was more successful and less time consuming than a broad approach. Fewer participants than anticipated were recruited, due to the difficulties encountered with site and interventionist start dates. However, we did not stop until we felt we had enough information to guide us when planning a larger trial. Recruitment via the JDR was not successful, due to large numbers of people indicating there were either suitable or erroneously believing they were in the catchment area, or unable get a hold of. Recruiting via the memory clinics were most successful. Attrition from the study due to ill health can be expected for this population, and four declined to give a reason. Of those who remained in the study, completion of intervention sessions was high.

All measures were acceptable to participants with only minor difficulties encountered. The TUG test occasionally required moving obstacles out of the way, but this was easily surmountable. Although there was some missing data, it was very low, less than $2 \%$, and this is to be expected. Although moderately lengthy at 90-120 minutes, all assessments were completed in one visit and no participants were distressed by the questions. With this limited sample, differences between groups were not expected, and were just looked at for exploratory purposes. Based on the results here, the authors conclude all of these measures would be suitable for the main trial.

The CDR proved to be challenging since only half the sites were using it regularly. As scores weren't already in the medical notes, they could not be used as a screening tool without seeking additional consent for its use. This would have raised the issue of potential participants having higher scores 
than the cut off for mild dementia. It was felt that to communicate this as a consequence of volunteering for a research study as opposed to being discussed as normal part of routine care was outside the remit of the study. Therefore an alternative, the MOCA, was used.

\section{Participant and facilitator qualitative perceptions of the PRIDE intervention}

The convenience sample was small representing only $11 \%$ of the dyads recruited to the feasibility study, however there was consistency in the accounts provided by person with dementia, supporters and facilitators. All found the intervention was an acceptable and positive experience. The timing of the intervention is an important factor with most relevance being with those newly diagnosed. Supporters and facilitators encouraged the person with dementia to act and reflect on activities and tasks. Having a facilitator seems to have empowered the person with dementia to be actively involved in the intervention. This maximised the ethos of the PRIDE study and contrasts with previous research which has found family members may try act in an over protective fashion and do things on behalf of the person (Sterin, 2002).

The personal interactions with the facilitator was reported as important. This may be a therapeutic alliance. Caution is needed as it is important to be able to differentiate whether positive outcome measures are due to the intervention or the therapeutic alliance between dyad and facilitator. This is especially important to explore as participants consistently reported they did not use the manual much between sessions. A larger trail should develop methods to explore this concept.

\section{Limitations}

Both the number of sites and participants were small, therefore we cannot comment on the potential efficacy of the intervention. It was however large enough to provide us with what we needed to adapt our methods for the RCT to ensure its success. In particular, the ability to supply intervention providers must rest with sites who can oversee their duties, workloads and contracts. Qualitive work was with an smaller number of dyads and intervention providers. The RCT will also include a qualitative component and our findings will help shape that, furthermore with a larger overall sample a larger cohort of participants will be finishing at any one time/in one location giving us a larger pool to recruit from. A larger sample size will also enable us to carry out suitable statistical analyses to test clinical diferences.

\section{Clinical and Research Implications}

Looking further into the mean differences in SMAS scores finding a moderate effect size indicates that with a larger sample significant differences are possible. These findings suggest that not only is the intervention beneficial in improving self management, but that this is especially true for those who would benefit the most. Self management skills, as measured by the SMAS, and the confidence to use them can be a key asset to limit the excess disability often found to have a significant impact on dementia (e.g., Brody et al 1971; Spector \& Orrell, 2010).

\section{Conclusions}

The PRIDE intervention proved to be acceptable to persons with dementia and their supporters, as shown by recruitment and retention rates, as well as quantitative feedback. Including memory clinic staff as intervention facilitators had a positive impact on getting sites up and running to take part in the study. Outcomes measure were suitable for use in our feasibility trial and with larger sample sizes differences pre and post intervention might be found. We believe it will be beneficial to evaluate PRIDE through a randomised control trial to investigate the efficacy of the intervention. 
References

Alzheimers Society. Dementia advisors: A cost effective approach to delivering integrated dementia care. 2016. Last accessed May 23, 2019.

https://www.google.com/url?sa=t\&rct=i\&q=\&esrc=s\&source=web\&cd=2\&ved=2ahUKEwjtmKCLxLHi AhVcUxUIHSrQBaIQFjABegQIBRAC\&url=https\%3A\%2F\%2Fwww.alzheimers.org.uk\%2Fsites\%2Fdefaul t\%2Ffiles\%2Fmigrate\%2Fdownloads\%2Fdementia advisers a cost effective approach to deliverin g integrated dementia care.pdf\&usg=AOvVaw2YiKdzhIMfS1Lv8GO1S7GM

Beecham J, Knapp M. Costing psychiatric interventions. In Measuring Mental Health Needs. G. Thornicroft editor. pp.163-183. London: Gaskell.1992

Birt et al 2019 Maintaining social connections in dementia a qualitative synthesis. In Qualitative Health Research In press

Borschmann, R, Patterson S, Poovendran, D, WilsonD, Weaver T. (2014) Influences on recruitment to randomised controlled trials in mental health settings in England: a national cross-sectional survey of researchers working for the Mental Health Research Network. MedicalResearch Methodology, $14 ; 23$.

Brody EM, Kleban MH, Lawton MP, Silverman HA. Excess disabilities of mentally impaired aged: impact of individualised treatment. Gerontologist, 1971; 11: 124-133.

Bucks RS, Ashworth DL, Wilcock GK, Siegfried K. Assessment of activities of daily living in dementia: development of the Bristol Activities of Daily Living Scale. Age Ageing. 1996;5(2): 113-120.

Bunn F, Goodman C, Sworn K, Rait G, Brayne C, Robinson L, McNeilly E, lliffe S. Psychosocial Factors That Shape Patient and Carer Experiences of Dementia Diagnosis and Treatment: A Systematic Review of Qualitative Studies. 2012. PLOS Medicine. https://doi.org/10.1371/journal.pmed.1001331

Byrne LM, Wilson P, Bucks RS, Hughes AO, Wilcock GK. The sensitivity to change over time of the Bristol Activities of Daily Living Scale in Alzheimer's disease. Int Journal Geriatric Psy. 2000; 5(7):656661.

Burgener S C, Buettner L L, Beattie E, Rose K M. Effectiveness of community-based, nonpharmacological interventions for early-stage dementia: Conclusions and recommendations. $J$ Gerontol Nurs. 2009; 35(3): 50-57. doi:10.3928/00989134-20090301-03

Clarke C L, Keyes, S E, Wilkinson H, Alexjuk J, et al. Healthbridge: The National Evaluation of Peer Support Networks and Dementia Advisers in implementation of the National Dementia Strategy for England. 2013. Retrieved from Department of Health and Social Care website: https://www.gov.uk/government/uploads/system/uploads/attachment data/file/246406/Demonstr ator Sites Evaluation - Final Report Master.pdf accessed February 262019

Coast J, Flynn TN, Natarajan L, Sproston K, Lewis J, Louviere JJ, Peters TJ. Valuing the ICECAP capability index for older people. Soc Sci Med.2008; 67(5): 874-882.

Craig P, Dieppe P, Macintyre S, Michie S, Nazareth I, \& Petticrew M. Developing and evaluating complex interventions: the new Medical Research Council guidance. BMJ Brit Med J. 2008; 337: a1655. 
Csipke, E., Yates, L., Moniz-Cook, E., Leung, P., Charlesworth, G., Walton, H., ... \&Orrell, M. Promoting Independence in Dementia (PRIDE): protocol for a feasibility trial of the PRIDE intervention for living well with mild dementia. Int J Clin Trials. 2018; 5 (4), 177-185.

Department for Constitutional Affairs. The Mental Capacity Act

2005. http://www.opsi.gov.uk/acts/acts2005/20050009.htm Accessed February 25, 2019.

Department of Health. Prime Minister's Challenge on Dementia 2020: Implementation plan. 2016. Retrieved from Department of Health and Social Care website: https://www.gov.uk/government/uploads/system/uploads/attachment data/file/507981/PM Dem entia-main acc.pdf Accessed February 25, 2019.

d'Orsi E, Xavier A J, Rafnsson S B, Steptoe A, et al. Is use of the internet in midlife associated with lower dementia incidence? Results from the English Longitudinal Study of Ageing. Aging Ment Health. 2017; 22 (11): 1525-1533.

Ellwood et al., (2018). Recruiting care homes to a randomised controlled trial. Trials 19:535.

European Union (EU) Joint Programme Neurodegenerative Diseases (JPND): JPND Research Strategy. 2012 http://www.neurodegenerationresearch.eu. Accessed 4 Nov 2017.

EuroQoL Group. EuroQoL: A new facility for the measurement of health related quality of life. Health Policy. 1990;16:199-208

Folstein MF, Folstein SE, McHugh PR. ("Mini-mental state". A practical method for grading the cognitive state of patients for the clinician. J Psychiatr Res, 1975;12(3):189-198.

Hogervorst E, Combrinck M, Lapuerta P, Rue J, Swales K, Budge M. The Hopkins verbal learning test and screening for dementia. Dementia GeriatrCogn.2002;13(1):13-20.

Hughes ME, Waite LJ, Hawkley LC, Cacioppo JT. A short scale for measuring loneliness in large surveys results from two population-based studies. Res Aging.2004;26(6):655-672.

Hyde M, Wiggins RD, Higgs P, Blane DB. A measure of quality of life in early old age: the theory, development, and properties of a needs satisfaction model (CASP-19). Aging Ment Health. 2003;7:186-194.

Jenson, E.A. \& Laurie, C. (2016) Doing real research: a practical guide to social research. London, Sage.

Katarzyna Małgorzata Lion, Dorota Szcześniak, Katarzyna Bulińska, Shirley Barbara Evans, Simon C. Evans, Francesca Lea Saibene, Alessia d'Arma, Elisabetta Farina, Dawn June Brooker, Rabih Chattat, Franka J. M. Meiland, Rose-Marie Dröes \& Joanna Rymaszewska (2019): Do people with dementia and mild cognitive impairments experience stigma? A cross-cultural investigation between Italy, Poland and the UK, Aging \& Mental Health.

Morris JC. The Clinical Dementia Rating (CDR): current version and scoring rules. Neurology. 1993;43(11): 2412-2414.

Mountain, G; Gossage-Worrall, R; Cattan, M; Bowling, A. Only available to a selected few? Is it feasible to rely on a volunteer workforce for complex intervention delivery? Health and Social Care in the Community (2017); 25 (1); 177-184. 
Nuno et al. (2017). Attitudes toward clinical trials across the Alzheimer's disease spectrum. Alzheimer's Research \& Therapy. 9:81.

Podsiadlo D, Richardson S. The timed" Up \& Go": a test of basic functional mobility for frail elderly persons. J Am Geratric Soc. 1991;39(2):142.

Rafnsson S B, Orrell M, d'Orsi E, HogervorstE, et al. Loneliness, social integration, and incident dementia over 6 years: prospective findings from the English Longitudinal Study of Ageing. $J$ Gerontol. 2017; Series B, gbx087, doi: 10.1093/geronb/gbx087

Reiflel B V, Larson E. Excess disability in dementia of the Alzheimer's type. In Light E, Lebowitz BDeditors Alzheimer's Disease Treatment and Family Stress. New York: Hemisphere; 1990.

Schuurmans H, Steverink N, Frieswijk N, Bunk BP, Slaets JP, Lindenberg S. How to measure selfmanagement abilities in older people by self-report. The development of the SMAS-30. Qual Life Re.2005;14(10):2215-2228.

Smith SC, Lamping DL, Banerjee S, Harwood A, Knapp M, et al. Measurement of health-related quality of life for people with dementia: Development of a new instrument (DEMQOL) and an evaluation of current methodology. Health Technol Assess. 2005;9(10):1-112.

Soni M, Orrell M, Bandelow S, Steptoe A, et al. Physical activity pre-and post-dementia: English Longitudinal Study of Ageing. Aging Men Health. 2017; 1-7.

doi.org/10.1080/13607863.2017.1390731

Spector A, Orrell M. Using a biopsychosocial model of dementia as a tool to guide clinical practice. International Psychogeriatrics, 2010; 22: 957-965.

Srivastava, A. \& Thomson, S. B. (2009).Framework Analysis: A Qualitative Methodology for Applied Policy Research. JOAAG, Vol. 4. No. 2

Sterin J. Essay on a word: a lived experience of Alzheimer's Disease. Dementia. 2002; 1: 7- 10.

Stoner, C.R., Orrell, M. Long, M., Csipke, E and Spector, A. The development and preliminary psychometric properties of two positive psychology outcome measures for people with dementia: the PPOM and the EID-Q. BMC Geriatr. 2017;17:72

DOI 10.1186/s12877-017-0468-6

Thabane, L, Ma J,Chu R,Cheng J,Ismaila A,Rios L, Robson L,Thabane M, Giangregorio L, GoldsmithC.A tutorial on pilot studies: the what, why and how. BMC Medical Research Methodology. 2010, 10:1.

Vertesi A, Lever JA, Molloy DW, Sanderson B, Tuttle I, Pokoradi L. Standardized mini-mental state examination. Use and interpretation. Can Fam Physician. 2001;47:2018-23.

Walton, H.J (2018). Evaluating the implementation of social interventions to improve independence in dementia (Doctoral Thesis (Ph.D). Available from UCL Discovery:

http://discovery.ucl.ac.uk/10064480/ 
Figure 1 Flow through feasibility study.

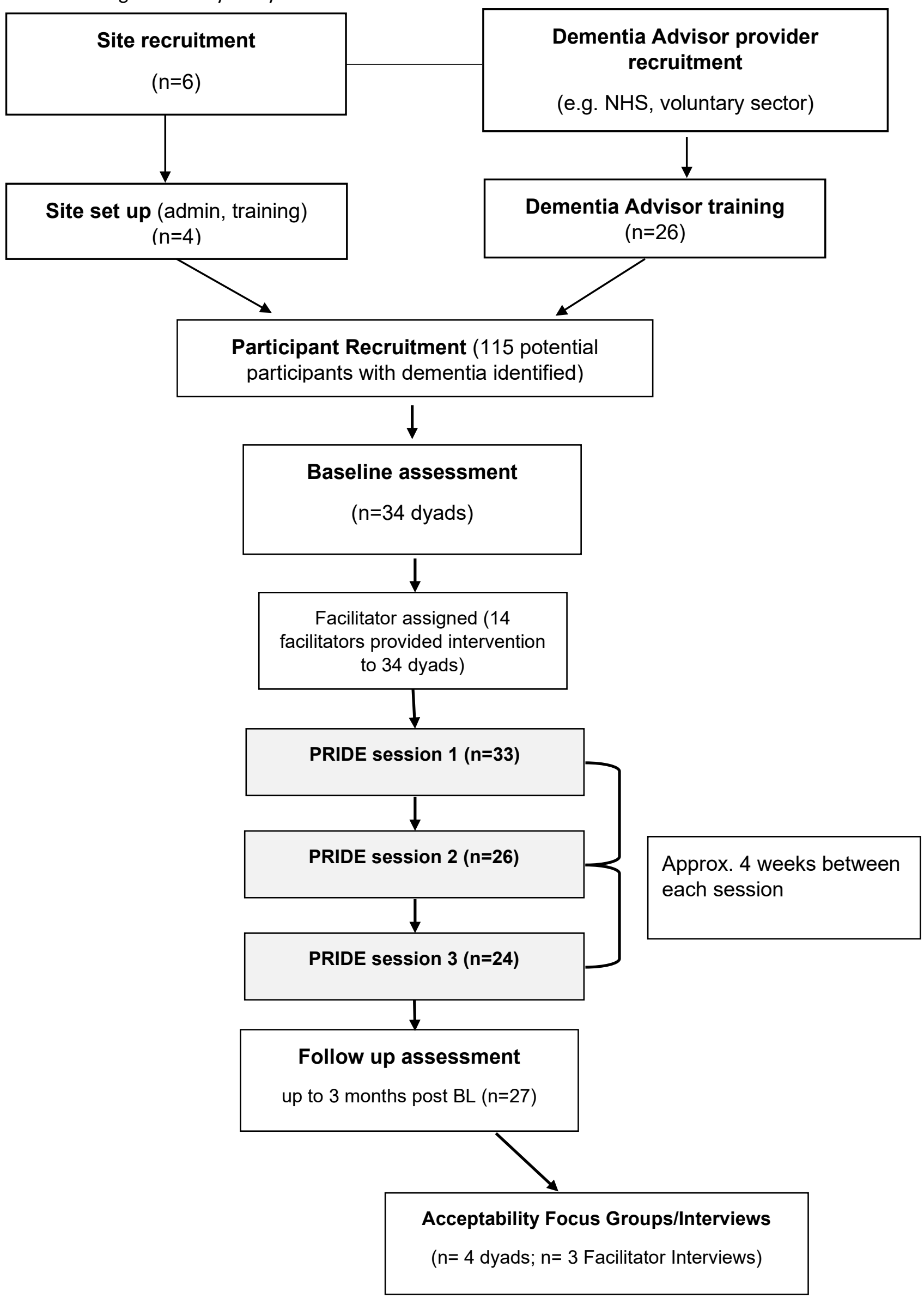


Table 1: Person with dementia baseline (BL) and follow up (FU)

\begin{tabular}{|c|c|c|c|c|c|c|c|c|}
\hline Questions & \multicolumn{3}{|c|}{ Baseline } & \multicolumn{3}{|c|}{ Follow Up } & T-test & $\begin{array}{l}\text { Significance } \\
\text { ( } 2 \text { tailed) }\end{array}$ \\
\hline $\begin{array}{l}\text { Control, autonomy, pleasure, } \\
\text { and self-realization (CASP 19) }\end{array}$ & 34 & 23.22 & 6.07 & 27 & 22.69 & 6.07 & .35 & .73 \\
\hline $\begin{array}{l}\text { EQ5-D (Health related quality of } \\
\text { life) }\end{array}$ & 34 & .80 & .23 & 27 & .81 & .23 & -.30 & .76 \\
\hline $\begin{array}{l}\text { Dementia Quality of Life } \\
\text { (DEMQOL) Total }\end{array}$ & 34 & 91.56 & 13.21 & 27 & 91.85 & 16.37 & -.08 & .94 \\
\hline $\begin{array}{l}\text { Engagement and Independence } \\
\text { in Dementia Questionnaire } \\
\text { (EID-Q) }\end{array}$ & 34 & 81.40 & 15.89 & 27 & 83.79 & 12.46 & -.64 & .52 \\
\hline $\begin{array}{l}\text { Self Management Abilities Scale } \\
30 \text { (SMAS 30) }\end{array}$ & 34 & 91.94 & 17.17 & 27 & 91.94 & 17.17 & -1.55 & .13 \\
\hline $\begin{array}{l}\text { Hopkins Verbal Fluency and } \\
\text { Learning Test (HVLT) Total }\end{array}$ & 34 & 11.09 & 4.6 & 27 & 12.00 & 5.2 & -.72 & .47 \\
\hline $\begin{array}{l}\text { Standardised Mini-Mental State } \\
\text { Examination (SMMSE) }\end{array}$ & 34 & 23.97 & 4.24 & 27 & 22.85 & 5.60 & .89 & .38 \\
\hline EQ5-D VAS & 34 & 70.97 & 18.50 & 27 & 74.56 & 18.83 & -.75 & .46 \\
\hline Timed Get up and Go (TUG) & 34 & 15.17 & 6.04 & 27 & 13.60 & 6.04 & 1.05 & .29 \\
\hline $\begin{array}{l}\text { ELSA: ELSA self-perceived social } \\
\text { connectedness }\end{array}$ & 34 & 5.65 & 1.43 & 27 & 5.68 & 4.21 & -.09 & .93 \\
\hline
\end{tabular}




\begin{tabular}{|l|c|c|c|c|c|c|c|c|}
\hline $\begin{array}{l}\text { (Dementia Quality of Life } \\
\text { (DEMQOL) Total * }\end{array}$ & 34 & 95.53 & 10.78 & 26 & 96.19 & 11.12 & .98 & .33 \\
\hline EQ5-D* & 34 & .65 & .23 & 26 & .67 & .23 & -.40 & .69 \\
\hline EQ5-D VAS* & 34 & 63.38 & 18.05 & 26 & 66.09 & 16.45 & .60 & .55 \\
\hline
\end{tabular}

* Note. Proxy measures. High scores indicate greater well being except on the IPA, EQ5-D and ELSA. 
Table2: Supporters baseline (BL) and follow up (FU)

\begin{tabular}{|l|c|c|c|c|c|c|c|}
\hline \multicolumn{1}{|c|}{ Questions } & Number & Mean & SD & Number & Mean & SD & $\begin{array}{l}\text { T-test } \\
\text { e (2 tailed) }\end{array}$ \\
\hline ICECAP-O & 34 & 13.47 & 3.06 & 26 & 14.54 & 3.29 & -1.30 \\
\hline EQ5-D & 34 & .74 & .24 & 26 & .78 & .22 & -.74 \\
\hline EQ5-D VAS & 34 & 76.35 & 13.58 & 26 & 81.66 & 12.24 & -1.56 \\
\hline
\end{tabular}

High scores on ICECAP-O scores indicate greater wellbeing, high scores on EQ5-D indicate lower wellbeing. 
Table 3. Enactment of the PRIDE intervention and SMAS scores. Post hoc exploration.

\begin{tabular}{|c|c|c|}
\hline & \multicolumn{2}{|c|}{ Mean SMAS } \\
\hline & Baseline & Follow up \\
\hline \multicolumn{3}{|c|}{ Self -reported Enactment Time $2^{*}$} \\
\hline $76-100 \%$ enactment & 97.64 (SD 15.60; $n=10)$ & 97.70 (SD 10.43; $\mathrm{n}=10)$ \\
\hline$\leq 75 \%$ enactment & 78.61 (SD 16.28; $n=9)$ & $96.42(S D 12.87 ; n=9)$ \\
\hline \multicolumn{3}{|c|}{ Self -reported Enactment Time 3} \\
\hline $76-100 \%$ & 97.58 (SD 18.73; $n=9)$ & $94.90(S D 16.03 ; n=9)$ \\
\hline$\leq 75 \%$ & 77.50 (SD 21.00; n= 5) & 96.35 (SD 13.82; n=5) \\
\hline Unknown & 92.94 (SD 16.07; $n=11)$ & 104.50 (SD 9.95; $n=4)$ \\
\hline
\end{tabular}

*Enactment was measured after interventions sessions 2 and 3 only.

Note. $\mathrm{N}^{\prime} \mathrm{s}$ in the unknown group are those who did not complete either the T2 or T3 enactment question

(For more details on the overall engagement scores see Walton et al, 2018). 